\title{
Estructura de la comunidad de cunáxidos (Acarina) edáficos de una selva baja caducifolia en Chamela, México
}

\author{
Blanca Estela Mejía-Recamier \& Gabriela Castaño-Meneses \\ Laboratorio de Ecología y Sistemática de Microartrópodos, Departamento de Ecología y Recursos Naturales, Facultad \\ de Ciencias, Universidad Nacional Autónoma de México, 04510 México D.F., México; bemr@hp.fciencias.unam.mx. \\ Tel: 56224902. Fax: 56224828
}

Recibido 19-X-2005. Corregido 24-XI-2006. Aceptado 14-V-2007.

\begin{abstract}
Community structure of edaphic cunaxids (Acarina) from a lowland dry forest in Chamela, México. The structure community of cunaxids mites was studied at a tropical dry forest in the Chamela Biological Station, Jalisco, Mexico. Monthly samples of litter and soil were taken during one year (July 1991-May 1992), in two small watersheds. A total of 43 species was recorded. We studied their abundance, species frequency (F), rareness and relative abundance (Ar), density (ind $\mathrm{m}^{-2}$ ), species richness (S), Shannon's diversity index (H'), and Pielou's evenness index (J'). The species richness was similar in litter and soil (38 species), but species composition was different. The higher density was found in soil, with $5074 \mathrm{ind} \mathrm{m}^{-2}$; litter density was $2199 \mathrm{ind} \mathrm{m}^{-2}$. The subfamilies with the highest density in both biotopes were Coleoscirinae $\left(\mathrm{H}=1127\right.$ ind m $\mathrm{m}^{-2} ; \mathrm{S}=2235$ ind m$\left.^{-2}\right)$ and Cunaxiinae $\left(\mathrm{H}=887\right.$ ind $\mathrm{m}^{-2} ; \mathrm{S}=1936$ ind $\left.\mathrm{m}^{-2}\right)$. The genus Pulaeus had the most species, and all were new to science. Mean and variance indicate an aggregate distribution for these cunaxids. By permanency and relative abundance, the dominant species were: Cunaxa potchensis, Coleoscirus simplex, Armascirus harrisoni, Cunaxoides sp. nov. 1, Cunaxoides sp. nov. 2, Pulaeus sp. nov. 15 and Pulaeus sp. nov. 16. An significant effect of the month and the biotope were recorded on the diversity and composition of cunaxid communities. Most of the species presented a significant effect of the month (24 spp.) and biotope (23 spp.), and five species only had a significant effect of the watersheds. The highest similarity was in litter (75\%) and soil (74 \%). The highest temporal variation in density, diversity and species richness was from April through June in litter, and from September through November in soil. The evenness was constant in both biotopes. In the dry period the soil presented higher values of density, while in the litter it was in the rainy season. The species with high soil density (FebruaryJune: dry season) in both watersheds, were: C. simple, C. potchensis, Cunaxa sp. nov. 1, Cunaxa sp. nov. 2, C. veracruzana, Dactyloscirus sp. nov. 2, A. harrisoni, Cunaxoides sp. nov. 1, Cunaxoides sp. nov. 2, P. pectinatus, Pulaeus sp. nov. 14, Pulaeus sp. nov. 15 and Pulaeus sp. nov. 16. They were the most representative species in the soil and litter of Chamela communities. The most abundant dry season species were C. potchensis in the soil and A. harrisoni in the litter. Rev. Biol. Trop. 55 (3-4): 911-930. Epub 2007 December, 28.
\end{abstract}

Key words: cunaxids, diversity, species richness, temporal variation, soil, litter.

En el ambiente edáfico, cerca del $90 \%$ de las poblaciones de microartrópodos está compuesta por colémbolos y ácaros, dentro de este último grupo, los oribátidos (Cryptosotigmata) y los astigmados son principalmente de hábitos saprofitos y micofagos, mientras que los prostigmados y mesostigmados son en su mayoría depredadores. Los prostigmados es el segundo grupo de importancia en el suelo en cuanto a su abundancia, después de los oribátidos.
En trabajos realizados en distintos ambientes, incluyendo zonas semiáridas, selvas bajas, bosques, suelos arenosos y de cultivo, se ha citado que el $50 \%$ del total de ácaros está compuesto por prostigmados (Estrada y Sánchez 1986, Moreno-Moreno 1996, De la Garza 2003). En las comunidades del suelo y hojarasca se han encontrado a 60 familias, 681 géneros y 6400 especies de prostigmados; los grupos de depredadores comprenden 26 familias, 
251 géneros y 1200 especies (Kethley 1990, Vázquez Rojas 2002). Los ácaros cunáxidos, prostigmados depredadores, constituyen un grupo de microartrópodos de vida libre de gran interés faunístico. Estos ácaros son capaces de explotar numerosos microhábitats, por lo que se les recolecta tanto en medios húmedos como en secos. Debido a su actividad como depredadores, son de gran importancia en las cadenas alimenticias de los medios edáficos y otros ambientes, siendo sus presas artrópodos de talla pequeña, principalmente ácaros de distintos Ordenes y colémbolos. En los últimos años se ha manifestado el papel significativo que desempeñan los depredadores en la densidad de las poblaciones, por lo que se les considera de gran importancia en la estructura de las comunidades del suelo y en el control biológico de muchas plagas agrícolas de ácaros, principalmente de los ácaros fitófagos (Muma 1960, Schruft 1971, Kethley 1982, May 2001). La densidad de los depredadores es paralela con la de sus presas, por lo que el grado en que los organismos se desarrollan, va a depender de las características propias de los individuos y los factores abióticos del suelo, tales como la temperatura, la humedad, el $\mathrm{pH}$, la porosidad y la disponibilidad de alimento. A su vez, hay cadenas alimenticias bien definidas y una intensa competencia por la supervivencia, que da como resultado que estos factores (bióticos y abióticos), influyan de manera considerable en la distribución espacial de los organismos del suelo. Esto permite utilizar a la fauna edáfica como bioindicadores para saber las condiciones en que se encuentran los suelos (Van Straalen y Verhoef 1997).

Sobre la ecología de los cunáxidos en particular, existen dos trabajos, uno realizado en Polonia por Michocka (1987), donde cita la distribución, así como aspectos taxonómicos de algunas especies de los géneros Bonzia, Cunaxa, Cunaxoides, Neocunaxoides, Scirula y Dactyloscirus.

En otro trabajo, realizado por Walter y Kaplan (1991), se estudia el tipo de alimentación de los géneros Pulaeus, Cunaxa y Dactyloscirus.
Los estudios realizados en México sobre los Cunaxidae, se han enfocado principalmente en aspectos taxonómicos (Baker y Hoffmann 1948, Smiley 1992). Uno de los estudios recientes fue el llevado a cabo por Vázquez Rojas (2002), en el cual estudia a los Prostigmata edáficos en la zona litoral del Golfo de México. Este trabajo menciona que los cunáxidos presentan un porcentaje de riqueza alto, y las especies que registra para una selva baja en esta zona fueron: Cunaxa setirostris, Cunaxa womersleyi, Dactyloscirus fixus, Dactyloscirus bison, Neocunaxoides andrei, Pulaeus americanus y Pulaeus franciscae.

El propósito del presente estudio es aportar datos sobre la estructura de cuatro comunidades de cunáxidos de una selva baja caducifolia en las costas del Pacífico mexicano, y las variaciones espaciales y temporales que presenta.

Área de estudio: la Estación de Biología Chamela (EBCH), UNAM, se localiza en el Municipio de Chamela, en la costa del Estado de Jalisco $\left(19^{\circ} 29^{\prime}\right.$ y $19^{\circ} 32^{\prime} \mathrm{N}$; 104 ${ }^{\circ} 58^{\prime}$ y $\left.105^{\circ} 05^{\prime} \mathrm{W}\right)$. La vegetación dominante en el área es el bosque tropical caducifolio (Bullock 1988).

Su topografía es muy irregular, lo que hace que gran parte de la zona se clasifique como lomeríos bajos que van de los 20 a los $250 \mathrm{~m}$. El clima de Chamela, de acuerdo a la clasificación de Köppen modificada por García (1988), es $A_{w_{0}} \mathrm{i}$, que corresponde a un clima cálido subhúmedo, con una marcada estacionalidad, siendo el más seco de los tipos subhúmedos. La precipitación promedio anual es de 788 $\mathrm{mm}$, con una gran variación interanual, por ejemplo, en 1992 se registró una precipitación de $1393 \mathrm{~mm}$, como resultado del efecto de los ciclones tropicales en la parte central de la costa del Pacífico, cambiando con ello los patrones de precipitación generalmente registrados (García-Oliva et al. 2002). La dinámica de las lluvias delimita dos estaciones climáticas: la de sequía, que abarca de diciembre hasta principios de junio; y la húmeda, que va de julio a octubre, siendo máxima precipitación agosto y septiembre (Bullock 1986), aunque se 
presentan algunas lluvias ocasionadas por los huracanes de diciembre a febrero. La temperatura media anual es de $24.6{ }^{\circ} \mathrm{C}$ y la humedad relativa es de $65 \%$ durante todo el año (GarcíaOliva et al. 2002).

Las comunidades vegetales dominantes son la selva baja caducifolia, con árboles de entre 5 y 12 m de altura, de copas extendidas lateralmente (que en época de sequía pierden la totalidad de sus hojas) y gran riqueza de especies. Se registra la existencia de más de 1036 especies de plantas vasculares, las dos familias más representativas son: Leguminosae (57 especies) y Euphorbiaceae (26 especies). Existen más de 227 especies de árboles, además de arbustos (216 spp.), lianas y trepadoras (187 spp.), hierbas (366 spp.) y epífitas (47 spp.); entre los más importantes destacan: Guapira linereaobractecta, Plumeria rubra, Bursera inestabilis, Lonchocarpus eriocarinalis y Celaeodedron mexicanum (Lott y Atkinson 2002).

Los tipos de suelos dominantes son los regosoles luvieútricos y èutricos, cambisoles éutricos y lixisol háplico (Cotler et al. 2002). Los suelos en la mayor parte de la estación son someros, su color oscila entre pardo amarillento pálido, en seco, y pardo amarillento oscuro, en húmedo. El promedio anual de humedad en el suelo es de $4.05 \%$. La textura es migajón arenoso, con bajo contenido de arcilla, y el porcentaje de porosidad es de $53 \%$. La proporción de las partículas en promedio es $62 \%$ arena, 20 $\%$ arcilla y $18 \%$ limo. El contenido de materia orgánica es bajo y el pH ácido. Es bajo el contenido de nutrientes, pero el calcio es el más abundante, seguido del magnesio y el potasio, y la producción de hojarasca es similar en ambas cuencas (Gómez -Anaya 1998).

\section{MATERIALES Y MÉTODOS}

Recolecta: la zona de estudio comprende un área de 10000 ha, integrada por un sistema de cinco cuencas hidrológicas, de las cuales se seleccionaron para el presente estudio, las cuencas denominadas 1 y 4 , por ser similares en cuanto al tamaño y productividad, con un promedio anual de hojarasca de $7642 \mathrm{~kg} \mathrm{ha}^{-1}$ (Cervantes et al. 1988, Martínez- Yrizar et al. 1996, Patiño 1990). En cada una se delimitó una superficie de $50 \times 50 \mathrm{~m}^{2}$. Dentro de esta área se seleccionaron puntos aleatoriamente para tomar muestras de suelo y hojarasca. Se hicieron muestreos mensuales de julio de 1991 a junio de 1992. En cada mes se tomaron en cada una de las cuencas, 10 muestras de hojarasca codificadas como $\mathrm{HC} 1$ y $\mathrm{HC} 4$, para las cuencas 1 y 4 respectivamente, y 10 de suelo (SC1 y SC4), para este último biotopo se utilizó un sacabocados con una profundidad de $5 \mathrm{~cm}$ y un diámetro de $11 \mathrm{~cm}\left(95 \mathrm{~cm}^{2}\right)$, la hojarasca tuvo un volúmen variado. Las colectas se realizaron entre las 07:00 a 11:00 hrs.

Las muestras recogidas en el campo se pusieron en cajas de plástico para ser trasladadas al laboratorio, ubicado en la Estación de Biología Chamela, el mismo día de su recolecta. La extracción de la fauna se realizó mediante embudos de Berlese-Tullgren, en donde las muestras permanecieron por seis días, los tres primeros a temperatura ambiente y los últimos, con luz artificial de un foco de $25 \mathrm{~W}$. La fauna se recolectó en frascos con alcohol al $96 \%$. Posteriormente el material obtenido se separó y cuantificó con la ayuda del microscopio estereoscópico. Para la elaboración de las preparaciones permanentes de los Cunaxidae, los ejemplares se aclararon en lactofenol por cinco días a una temperatura de $40{ }^{\circ} \mathrm{C}$; posteriormente se hicieron las preparaciones en líquido de Hoyer.

La determinación a nivel de especie se realizó bajo el microscopio de contraste de fases, con la ayuda de literatura especializada del grupo. El material estudiado se encuentra depositado en la Colección de ácaros del Laboratorio de Ecología y Sistemática de Microartrópodos, de la Facultad de Ciencias, UNAM.

Análisis de datos: Para cada comunidad de cunáxidos se determinó la abundancia relativa (\%), densidad (ind $\mathrm{m}^{-2}$ ), Frecuencia $(\mathrm{F})$, porcentaje de permanencia (Pp), así como los índices de diversidad de Shanon $\left(\mathrm{H}^{\prime}\right)$, índice de equitatividad de Pielou (J'), el índice 
de dominancia de Simpson $(\lambda)$, la similitud de Sörensen y los números de Hill (N2), de acuerdo con Ludwing y Reynols (1988). Para comparar entre los índices de diversidad de Shanon, se realizó una prueba de t modificada (Zar 1984).

El efecto de la cuenca, el biotopo y recolecta se evaluó mediante un análisis de varianza múltiple (MANOVA), realizando las comparaciones post hoc, mediante la prueba de Tukey. Para la normalización de los datos se utilizó una corrección de tipo $\sqrt{x+0.5}$ (Zar 1984). Las pruebas estadísticas se realizaron con el programa estadístico STATISTICA ver. 6.0 (StatSoft Inc. 1995).

\section{RESULTADOS}

Riqueza y composición: Se recolectaron un total de 4720 cunáxidos en el suelo y hojarasca en la Estación de Biología Chamela. El suelo presentó la mayor abundancia, con un

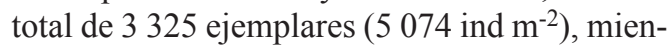
tras que en la hojarasca fue de 1395 (2 199 ind $\mathrm{m}^{-2}$ ). Tanto en las comunidades del suelo, como en las de hojarasca, la abundancia relativa y densidad fue más similar entre los biotopos, que entre cuencas (Cuadro 1), para el SC1 fue de $37 \%$; para el SC4 de $34 \%$; en HC1 de 14 $\%$ y en HC4 de $15 \%$ (Cuadro 1).

De acuerdo con el MANOVA, no se encontró efecto significativo de la cuenca, ni de ninguna de sus interacciones, sobre la abundancia de los cunáxidos. Sin embargo, el biotopo $(\mathrm{F}=27.05, \mathrm{gl}=1.432, \mathrm{p}<0.0005) \mathrm{y}$ el mes $(\mathrm{F}=2.81, \mathrm{gl}=11.432, \mathrm{p}<0.005)$ si tuvieron un efecto significativo (Cuadro 2). El análisis post hoc mediante la prueba de Tukey mostró que las diferencias se encuentran entre febrero, mayo y junio (Fig. 1).

La riqueza que presentaron los cunáxidos en Chamela fue de 43 especies en total. En cuanto a la riqueza en la hojarasca y el suelo, en general fue similar, sin embargo, al estudiar por separado las cuatro comunidades, se encontró que en el $\mathrm{SC} 1$ y en la $\mathrm{HC} 1$ fue mayor la riqueza (Cuadro 1).
De la revisión de los ejemplares de la Familia Cunaxidae se identificaron cuatro Subfamilias, de éstas, las que presentaron una mayor densidad fueron Coleoscirinae $(\mathrm{H}=1$ 127 ind $\mathrm{m}^{-2} ; \mathrm{S}=2235$ ind $\mathrm{m}^{-2}$ ) y Cunaxiinae $\left(\mathrm{H}=887\right.$ ind $\mathrm{m}^{-2} ; \mathrm{S}=1936$ ind $\left.\mathrm{m}^{-2}\right)$. De los 10 géneros identificados en este trabajo, los que tuvieron un mayor número de especies fueron: Pulaeus con 18 especies, de las cuales 17 se considera son nuevas especies para la ciencia, su densidad fue de 887 ind $\mathrm{m}^{-2}$ en la hojarasca y 1936 ind $\mathrm{m}^{-2}$ en el suelo; Cunaxa con 7 (incluyendo 5 especies nuevas para la ciencia), con una densidad de: $\mathrm{H}=530$ ind $\mathrm{m}^{-2}$ y $\mathrm{S}=1253$ ind $\mathrm{m}^{-2}$; Dactyloscirus con 6 especies, todas consideradas nuevas para la ciencia y con una densidad de $\mathrm{H}=307$ ind $\mathrm{m}^{-2} \mathrm{y} \mathrm{S}=536$ ind $\mathrm{m}^{-2}$, Cunaxoides y Neoscirula con 3 especies nuevas para la ciencia cada una y una densidad $\mathrm{H}=$ 232 ind $\mathrm{m}^{-2} \mathrm{y} \mathrm{S}=696$ ind $\mathrm{m}^{-2} \mathrm{y} \mathrm{H}=30$ ind $\mathrm{m}^{-2} \mathrm{y}$ $\mathrm{S}=125$ ind $\mathrm{m}^{-2}$ respectivamente (Fig. 2A-D).

En la fauna del suelo se ha observado que las poblaciones tienden a una distribución agregada, en este trabajo se encontró que los cunáxidos presentaron tal distribución, ya que su varianza siempre fue mayor que la media en cada una de las comunidades estudiadas. Así mismo, su distribución fue muy heterogénea y se observaron especies con muchos ejemplares y pocas especies con muy pocos representantes en el período de recolecta (Fig. 2E).

La composición de las comunidades fue diferente, ya que se encontraron tres especies exclusivas en el suelo Neoscirula sp. nov. 1, Neoscirula sp. nov. 3 y Pulaeus sp. nov. 5; y una para la hojarasca: Pulaeus sp. nov. 6; pero al estudiar cada una de las comunidades se observó como exclusivas en SC1: Cunaxa sp. nov. 5 y Pulaeus sp. nov. 2; en HC1: Pulaeus sp. nov. 3, Pulaeus sp. nov. 4 y Pulaeus sp. nov. 17; y por último en HC4 Pseudobonzia sp. nov. 1 (Cuadro 1).

Abundancia relativa (Ar), coeficiente de frecuencia $(\mathrm{F})$ y Porcentaje de permanencia: a partir de los valores obtenidos con el porcentaje de permanencia y abundancia relativa, se observó que las especies dominantes tanto en el suelo y como en la hojarasca, en ambas 


\section{CUADRO 1}

Densidad, abundancia absoluta y composición de los Cunaxidae por comunidad, en la EBCh en Chamela, Jalisco. Suelo cuenca 1 (SC1); Suelo cuenca 4 (SC4); Hojarasca cuenca 1 (HC1); Hojarasca cuenca 4 (HC4); índice de diversidad de Shannon $\left(H^{\prime}\right)$; dominancia de Simpson $(\lambda)$; número de Hill (N2); equitatividad ( $\left.J^{\prime}\right)$.

TABLE 1

Density, absolute abundance and composition of the Cunaxidae community, in the EBCh in Chamela, Jalisco. Litter watershed 1 (HC1); litter watershed 4 (HC4); soil watershed 1 (SC1); soil watershed 4 (SC4); Shannon's index of diversity ( $H$ '); dominance of Simpson $(\lambda)$; number of Hill (N2); Pielou's evenness index $(J ')$

Especie / Comunidad

Neoscirula sp nov. 1

2 Neoscirula sp nov. 2

$3 \quad$ Neoscirula sp nov. 3

4 Scutascirus sp nov. 1

5 Coleoscirus simplex

$6 \quad$ Pseudobonzia sp nov. 1

$7 \quad$ Pseudobonzia sp nov. 2

$8 \quad$ Cunaxa potchensis

9 Cunaxa veracruzana

10 Cunaxa sp nov. 1

11 Cunaxa sp nov. 2

12 Cunaxa sp nov. 3

13 Cunaxa sp nov. 4

14 Cunaxa sp nov. 5

15 Dactyloscirus sp nov. 1

16 Dactyloscirus sp nov. 2

17 Dactyloscirus sp nov. 3

18 Dactyloscirus sp nov. 4

19 Dactyloscirus sp nov. 5

20 Dactyloscirus sp nov. 6

21 Armascirus harrisoni

22 Cunaxoides sp nov. 1

Cunaxoides sp nov. 2

4 Cunaxoides sp nov. 3

Pulaeus sp nov. 1

Pulaeus sp nov. 2

Pulaeus sp nov. 3

Pulaeus sp nov. 4

$30 \quad$ Pulaeus sp nov. 5
$\mathrm{HC}$

0

9

0

19

18

0

\section{1}

66

46

20

30

15

4

0

16

35

2

14

8

18

99

48

5 Neocunaxoides sp nov. 1

$\begin{array}{lllll}\mathrm{HC} & \mathrm{SC} 1 & \mathrm{SC} 4 & \text { Hojarasca } & \text { Suelo }\end{array}$

(10)

3

4

0

17

0

31

47

8

17

111

84

28

82

17

14

0

$$
21
$$

78

18

26

34

18

184

119

26

2

6

5

0

1

8

0 
CUADRO 1 (Continuación)

Densidad, abundancia absoluta y composición de los Cunaxidae por comunidad, en la EBCh en Chamela, Jalisco. Suelo cuenca 1 (SC1); Suelo cuenca 4 (SC4); Hojarasca cuenca 1 (HC1); Hojarasca cuenca 4 (HC4); índice de diversidad de Shannon ( $\left.H^{\prime}\right)$; dominancia de Simpson ( $\lambda$ ); número de Hill (N2); equitatividad ( $\left.J^{\prime}\right)$.

TABLE 1 (Continued)

Density, absolute abundance and composition of the Cunaxidae community, in the EBCh in Chamela, Jalisco. Litter watershed 1 (HC1); litter watershed 4 (HC4); soil watershed 1 (SC1); soil watershed 4 (SC4); Shannon's index of diversity ( $H$ '); dominance of Simpson ( $\lambda)$; number of Hill (N2); Pielou's evenness index $(J$ ')

\begin{tabular}{|c|c|c|c|c|c|c|}
\hline Especie / Comunidad & $\mathrm{HC} 1$ & $\mathrm{HC} 4$ & $\mathrm{SC} 1$ & $\mathrm{SC} 4$ & Hojarasca & Suelo \\
\hline Pulaeus sp nov. 6 & 1 & 8 & 0 & 0 & 9 & 0 \\
\hline Pulaeus sp nov. 7 & 1 & 0 & 3 & 5 & 1 & 8 \\
\hline Pulaeus sp nov. 8 & 8 & 20 & 10 & 40 & 28 & 50 \\
\hline Pulaeus sp nov. 9 & 11 & 20 & 47 & 8 & 31 & 55 \\
\hline Pulaeus pectinatus & 32 & 16 & 60 & 14 & 48 & 74 \\
\hline Pulaeus sp nov. 10 & 9 & 12 & 6 & 16 & 21 & 22 \\
\hline Pulaeus sp nov. 11 & 9 & 0 & 26 & 0 & 9 & 26 \\
\hline Pulaeus sp nov. 12 & 0 & 5 & 0 & 7 & 5 & 7 \\
\hline Pulaeus sp nov. 13 & 8 & 0 & 9 & 29 & 8 & 38 \\
\hline Pulaeus sp nov. 14 & 19 & 27 & 52 & 43 & 46 & 95 \\
\hline Pulaeus sp nov. 15 & 7 & 43 & 32 & 78 & 50 & 110 \\
\hline Pulaeus sp nov. 16 & 71 & 58 & 156 & 69 & 129 & 225 \\
\hline Pulaeus sp nov. 17 & 11 & 0 & 0 & 0 & 11 & 0 \\
\hline Abundancia Total & 682 & 713 & 1658 & 1581 & 1395 & 3239 \\
\hline Densidad (ind $\mathrm{m}^{-2}$ ) & 1082 & 1117 & 2597 & 2472 & 2199 & 5074 \\
\hline Riqueza & 35 & 30 & 37 & 33 & 38 & 38 \\
\hline $\mathrm{H}^{\prime}$ & 2.07 & 2.1 & 2.16 & 2.15 & 3.29 & 3.3 \\
\hline$\lambda$ & 0.74 & 0.70 & 0.92 & 0.92 & 0.14 & 0.95 \\
\hline J' & 0.58 & 0.59 & 0.60 & 0.63 & 0.89 & 0.96 \\
\hline $\mathrm{N} 2$ & 13 & 14 & 11 & 11 & 7 & 11 \\
\hline
\end{tabular}

cuencas, fueron: $C$. simples, $C$. potchensis, $A$. harrisoni y Pulaeus sp. nov. 16. Es importante señalar que, las tres primeras especies, tuvieron un $100 \%$ de porcentaje de permanencia en el suelo (Fig. 3 y 4 )

Por otra parte, Cunaxoides sp. nov. 2, Scutascirus sp. nov. 1 y Cunaxa veracruzana se les encontró como dominantes en el suelo de ambas cuencas; mientras que a Cunaxoides sp. nov. 1 y Dactyloscirus sp. nov. 2, lo fueron en la hojarasca (Fig. 3).
Por otro lado, las especies poco dominantes y abundantes en ambos biotopos fueron: Cunaxa sp. nov. 2, Pulaeus pectinatus, Dactyloscirus sp. nov. 5, Pulaeus sp. nov. 14 y Pulaeus sp. nov. 15. Asimismo, Cunaxa sp. nov. 1, Cunaxa sp. nov. 3, Dactyloscirus sp. nov. 1 y Dactyloscirus sp. nov. 6, lo fueron sólo para la hojarasca (Fig. 3 y 4). El resto las especies fueron raras, con un promedio de porcentaje de permanencia del $13 \%$.

El valor obtenido de la frecuencia en todas las especies fue accidental en la hojarasca y 
CUADRO 2

MANOVA, para la densidad total de los cunáxidos encontrados en el suelo y hojarasca en las cuencas 1 y 4 en Chamela, Jalisco. (n.s $=$ no significativo; $*=p<0.05$ significativo; $* * p<0.005 ; * * *=p<0.0005$ )

TABLE 2

MANOVA, total density of the cunaxids found in the litter and soil in the watersheds 1 and 4 in Chamela, Jalisco. (n.s = not significant; $*=p<0.05$ significant; $* * p<0.005 ; * * *=p<0.0005$ )

$\begin{array}{ccccc}\text { Fuente } & \text { Gl } & \text { F } & p & \\ \text { C } & 1,436 & 0.94 & 0.33 & \text { n.s } \\ \text { B } & 1,432 & 27.05 & 0.000 & * * * \\ \text { M } & 11,432 & 2.81 & 0.002 & * * \\ \text { CxB } & 1,432 & 0.63 & 0.43 & \text { n.s } \\ \text { CxM } & 11,432 & 0.58 & 0.84 \\ \text { BxM } & 11,432 & 1.59 & 0.10 & \text { n.s } \\ \text { CxBхM } & 11,432 & 0.74 & 0.70 & \text { n.s }\end{array}$

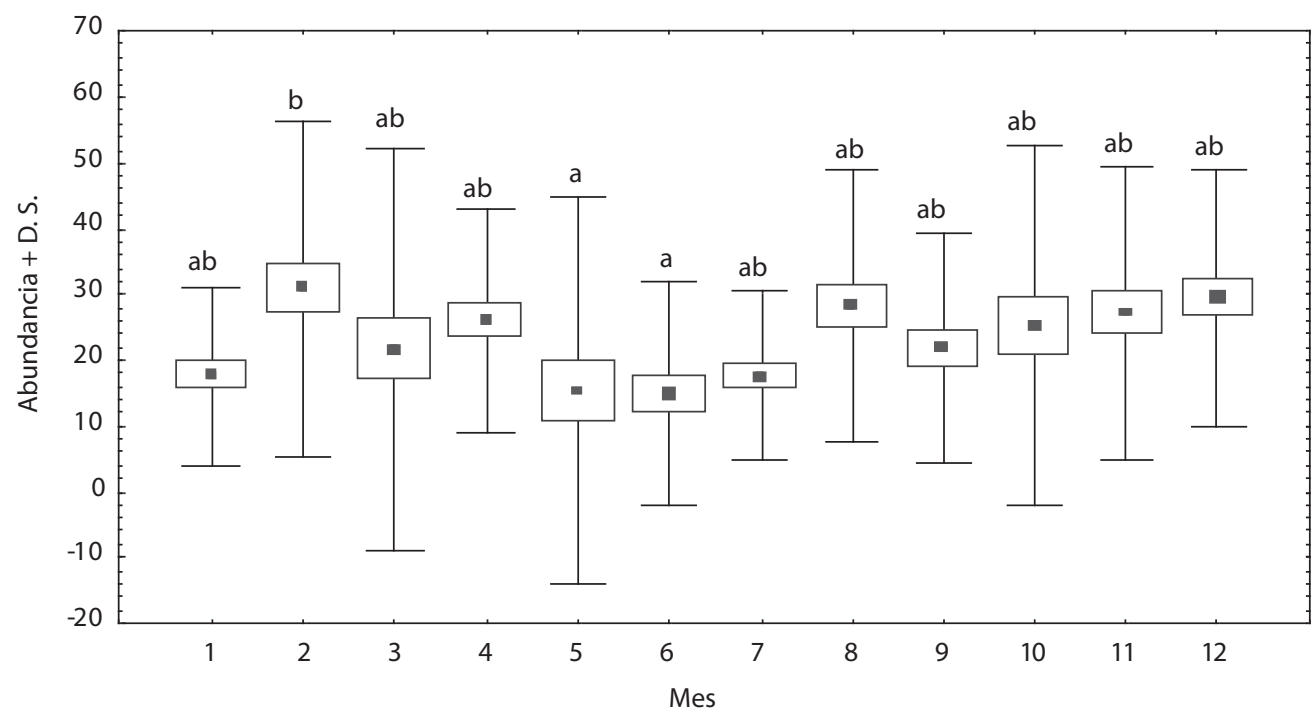

Fig. 1. Valores mensuales promedio de la abundancia de cunaxidos en suelo y hojarasca de la EBCH, Jalisco. Las letras distintas denotan diferencias significativas de acuerdo a la prueba de Tukey $(\mathrm{p}<0.05)$.

Fig. 1. Average monthly values for cunaxids abundance in litter and soil of the EBCH, Jalisco. Different letters denote significant differences among the months according, Tukey $(\mathrm{p}<0.05)$. 

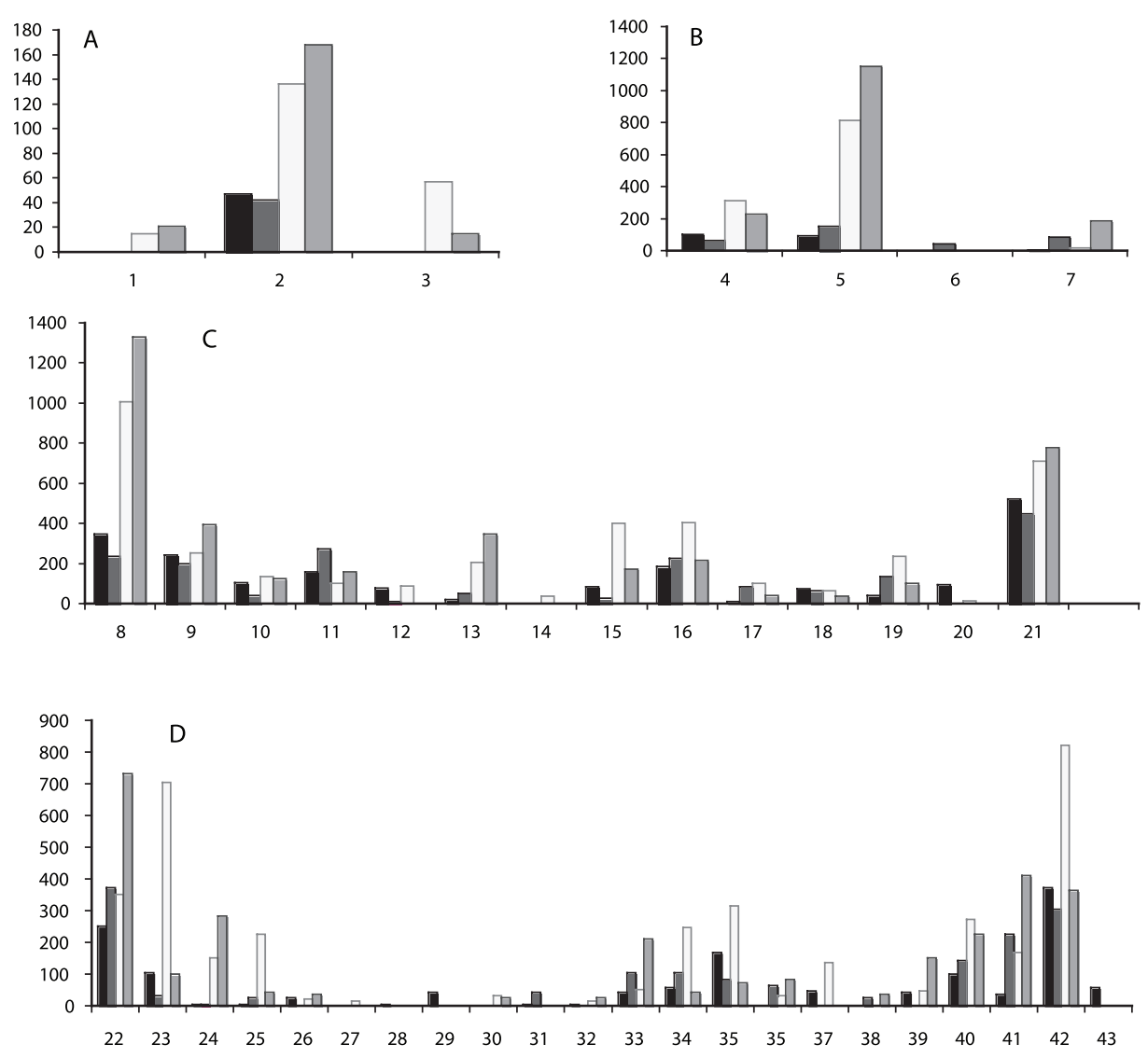

$\begin{array}{lll}\square \mathrm{HC} 1 & \square \mathrm{HC} 4\end{array} \mathrm{SC1} \quad \square \mathrm{SC4}$

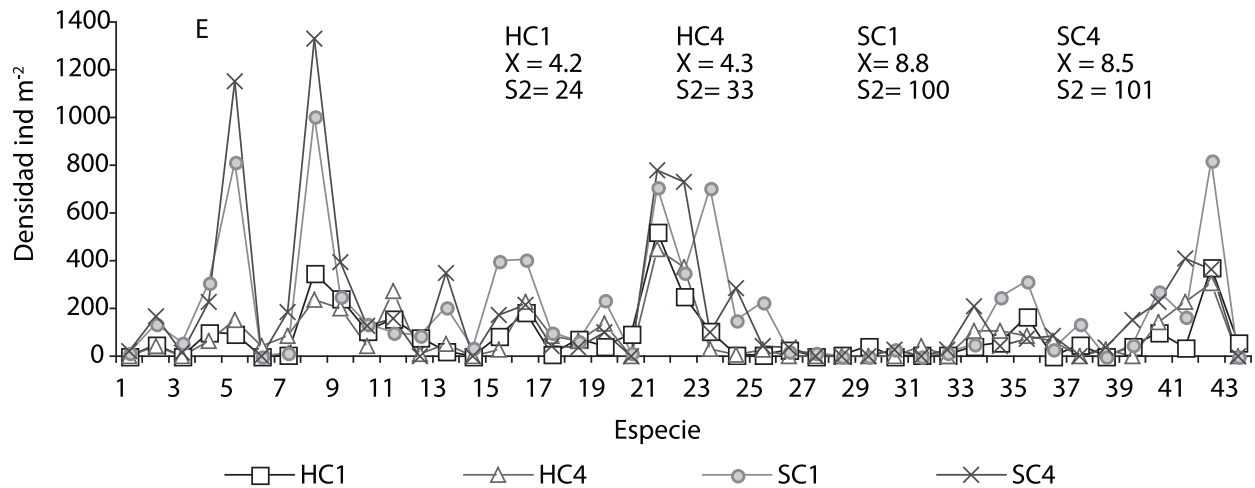

Fig. 2. Estructura de las Subfamilias de Cunaxidae en las comunidades de de la EBCH en Jalisco, México. A. Bonziinae; B. Coleoscirinae; C. Cunaxiinae; D. Cunaxoidinae; E. Comunidades de cunáxidos. Ver cuadro 1, para el nombre de las especies.

Fig. 2. Subfamily structure for Cunaxidae communities of the EBCH in Jalisco, Mexico. A. Bonziinae; B. Coleoscirinae; C. Cunaxiinae; D. Cunaxoidinae; E. Cunaxidae communities of Chamela. For cunaxid species names, see Table 1. 


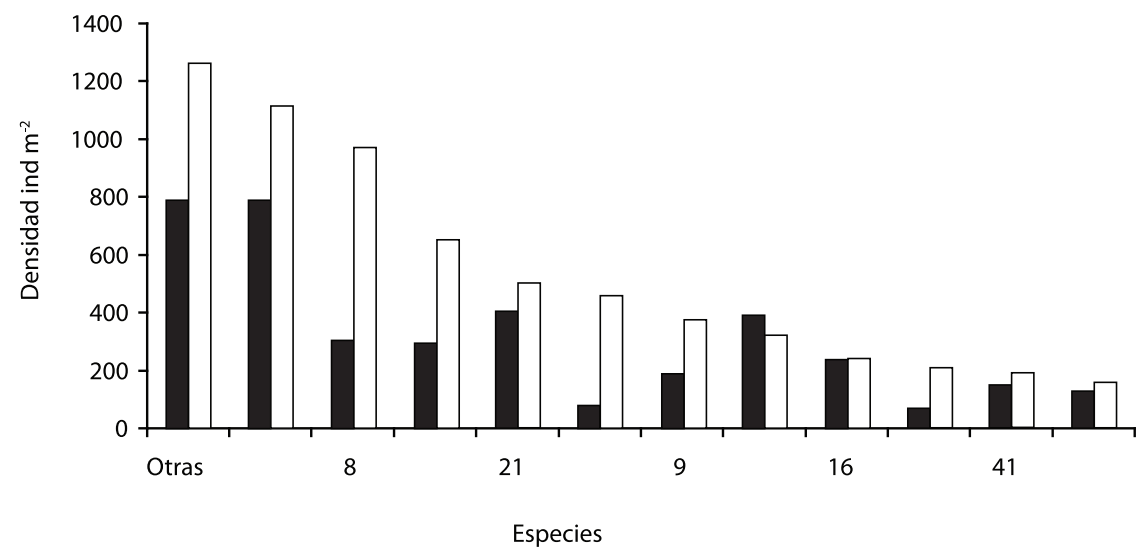

Hojarasca

$\square$ Suelo

Fig. 3. Densidad de las especies más abundantes en la comunidad de cunáxidos de suelo y hojarasca en las cuencas 1 y 4. 5=Coleoscirus simplex; 8=Cunaxa potchensis, 9=C. veracruzana, 15=Dactyloscirus $\mathrm{sp}$. nov. 1; 16=Dactyloscirus $\mathrm{sp}$. nov. 2; $21=$ Armascirus harrisoni; $22=$ Cunaxoides sp. nov. $1 ; 23=$ Cunaxoides sp. nov. 2; 40=Pulaeus sp. nov. 14; 41=Pulaeus sp. nov. $15 ; 42=$ Pulaeus sp. nov. 16 .

Fig. 3. Density of the most abundant cunaxids from soil an litter of watersheds 1 and $4.5=$ Coleoscirus simplex; $8=$ Cunaxa potchensis, $9=$ C. veracruzana, $15=$ Dactyloscirus sp. nov. $1 ; 16=$ Dactyloscirus sp. nov. 2; 21=Armascirus harrisoni; 22=Cunaxoides sp. nov. 1; 23=Cunaxoides sp. nov. 2; 40=Pulaeus sp. nov. 14; 41=Pulaeus sp. nov. 15; 42=Pulaeus sp. nov. 16.

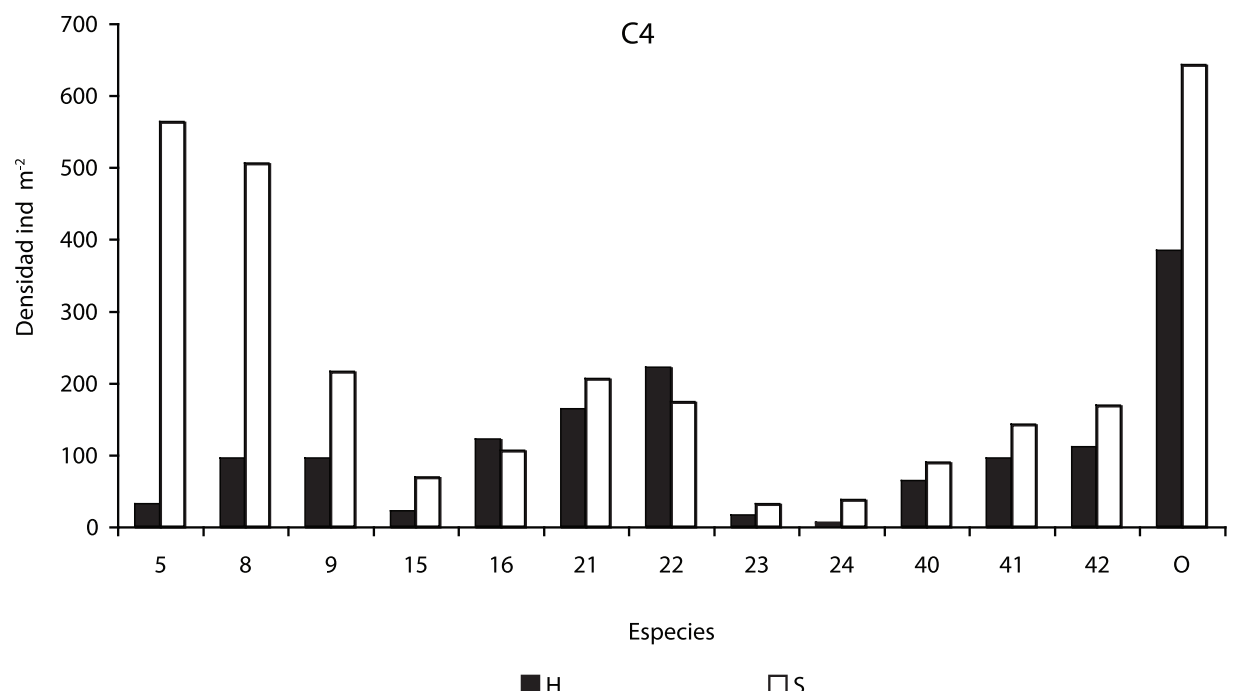

Fig. 4. Estructura de las cuatro comunidades de cunáxidos. $5=$ Coleoscirus simplex; $8=$ Cunaxa potchensis, $9=$ C. veracruzana, 15=Dactyloscirus sp. nov. 1; 16=Dactyloscirus sp. nov. 2; 21=Armascirus harrisoni; 22=Cunaxoides sp. nov. 1; 23=Cunaxoides sp. nov. 2; 40=Pulaeus sp. nov. 14; 41=Pulaeus sp. nov. 15; 42=Pulaeus sp. nov. 16; O, Otras especies con baja densidad.

Fig. 4. Structure of the four cunaxids communities in Chamela, Jal. $5=$ Coleoscirus simplex; $8=$ Cunaxa potchensis, $9=C$. veracruzana, 15=Dactyloscirus sp. nov. 1; 16=Dactyloscirus sp. nov. 2; 21=Armascirus harrisoni; 22=Cunaxoides sp. nov. 1; 23=Cunaxoides sp. nov. 2; 40=Pulaeus sp. nov. 14; 41=Pulaeus sp. nov. 15; 42=Pulaeus sp. nov. 16; O, Others species with low density. 
suelo de la selva baja caducifolia de Chamela, Jalisco.

Efecto de la cuenca (C), biotopo (B) y mes (M) sobre la abundancia de las especies de los cunáxidos de Chamela: el análisis de varianza para evaluar el efecto de la C, B, y M, sobre las abundancias de las especies de cunáxidos, mostró efectos diferenciales, como se puede ver en el Cuadro 3. En la mayoría de las especies se presenta un efecto significativo del biotopo (23 spp.) y mes (24 spp.), y sólo cinco especies se encontró un efecto significativo de la cuenca (Cuadro 3).

En las especies Cunaxa sp. nov. 3, Cunaxa sp. nov. 5, Dactyloscirus sp. nov. 4, Cunaxoides sp. nov. 2, Pulaeus sp. nov. 2, Pulaeus sp. nov. 3, Pulaeus sp. nov. 4 y Pulaeus sp. nov. 6, no se encontró ningún efecto significativo de las variables consideradas (C, B. M), ni de sus interacciones.

Diversidad (H'), Dominancia de Simpson $(\lambda)$ y equtatividad ( $J$ '), de la fauna de cunáxidos: de acuerdo al análisis de la prueba de t, parea el índice de diversidad, no se detectaron diferencias significativas entre los biotopos de ambas cuencas $(\mathrm{t}=0.30 ; \mathrm{p}>0.05 ; \mathrm{gl}=241)$.

Por lo anteriormente citado, se tomaron en cuenta los datos totales de la diversidad encontrada para las comunidades de suelo y hojarasca. La diversidad es similar en la hojarasca y suelo, así como la riqueza y equitatividad. Sin embargo, en el suelo hubo una mayor dominancia y densidad, por lo tanto un número mayor de especies muy abundantes (Cuadro 1), ésto se debe a que hay un número mayor de especies abundantes $\left(\mathrm{N}_{1}=16,11\right.$, suelo y hojarasca, respectivamente) y muy abundantes $\left(\mathrm{N}_{2}=17\right.$, 11), como son: $C$. potchensis, $C$. simplex, $A$. harrisoni Cunaxoides sp. nov. 1 y Pulaeus sp. nov. 16 (Cuadro 1). Todo lo contrario se observó en las comunidades de la hojarasca, ambas tiene menor diversidad, equitatividad, riqueza, diversidad $\left(H^{\prime}\right)$, dominancia $(\lambda)$, así como una mayor uniformidad $\left(\mathrm{J}^{\prime}\right)$; existe también una presencia menor de especies abundantes $(\mathrm{E} 5=0.77)$ y por lo tanto hay una mayor codominancia entre las especies.
Coeficiente de similitud faunística de Sörensen entre las comunidades de cunáxidos: el índice de Sörensen nos ayuda a determinar el posible cambio o efecto sobre la composición de las comunidades de cunáxidos. Los valores de similitud fueron mayores entre los biotopos de la hojarasca que los del suelo, como se puede ver en el Cuadro 4. En las comunidades de ambas cuencas tuvieron mucha similitud en su composición y comparten las mismas especies (Cuadro 1). Como se puede ver en el Cuadro 4, la mayor diferencia estuvo entre las comunidades de $\mathrm{HC} 1-\mathrm{SC} 4$, HC1-SC1 y HC4- SC1.

Variación temporal de la densidad, diversidad (H'), dominancia de Simpson $(\lambda)$ y equitatividad ( $J$ ') de la fauna de cunáxidos: se observaron variaciones tanto en la densidad como en la diversidad en el período de estudio, en el suelo y en la hojarasca. En general, en la hojarasca las mayores densidades se presentaron en febrero, octubre y noviembre; mientras que para el suelo fueron en marzo, mayo, agosto, octubre y diciembre. En la hojarasca los meses con mayor diversidad y riqueza fueron: febrero, octubre y noviembre; sin embargo en el suelo, sólo en agosto. La diversidad y riqueza se vio afectada por la densidad en las cuatro comunidades, siendo más notoria en las comunidades de hojarasca de marzo a junio, ésto debido a que es un sustrato sensible a los cambios climático. En ambos biotopos la equitatividad fue más constante en el transcurso del año.

En las comunidades de la hojarasca abril a julio mostraron una disminución en su diversidad, riqueza, densidad y un número menor de especies dominantes, sólo la equitatividad fue mayor durante todo el año, las poblaciones de cunaxidos fueron más homogéneas. Se incrementó el índice de dominancia de Simpson $(\lambda)$ en la $\mathrm{HCl}$ en febrero, junio, agosto, septiembre y octubre, las especies más dominantes fueron: Cunaxa sp. nov. 1, Cunaxa sp. nov. 2, Dactyloscirus sp. nov. 1, Cunaxoides sp. nov. 2 y Scutascirus sp. nov. 1. Mientras que en al hojarasca fueron abril y octubre con las 
CUADRO 3

MANOVA, para las especies de cunáxidos encontrados en el suelo y hojarasca en las cuencas 1 y 4 en Chamela, Jalisco. Cuenca (C); Biotopo (B); Mes (M). ( $g l, C, B, C x B=1,432 ; g l, M, C x M, B x M, C x B x M=11,432$; no significativo $=n$. s; significativo con un $*=p<0.05 ; * *=p<0.005 ; * * *=p<0.0005)$

TABLE 3

MANOVA, cunaxids species found in the litter and soil in the watersheds 1 and 4 in Chamela, Jalisco. watershed (C); Biotope (B); Month (M). (gl, C, B, CxB =1,432; gl, M, CxM, BxM, CxBxM=11, 432; not significant = n.s; significant with $a *=p<0.05 ; * *=p<0.005 ; * * *=p<0.0005$ )

\begin{tabular}{|c|c|c|c|c|c|c|c|c|c|c|c|c|c|c|}
\hline & $\mathrm{C}$ & & $\mathrm{B}$ & & M & & $\mathrm{CxB}$ & & $\mathrm{CxM}$ & & BxM & & CxBxM & \\
\hline Pseudobonzia sp. nov. 2 & 12 & $* *$ & 12 & $* * *$ & 2.46 & $* *$ & 8.2 & $* *$ & 1.3 & n.s. & 3 & $* *$ & 2.1 & $*$ \\
\hline Pulaeus sp. nov. 15 & 3.9 & $*$ & 4.9 & * & 3 & $* *$ & 0.5 & n.s. & 2.8 & $* *$ & 2.2 & $*$ & 1.8 & $*$ \\
\hline Dactyloscirus sp. nov. 5 & 0.1 & n.s & 8.4 & $* *$ & 6.7 & $* * *$ & 0.2 & n.s. & 3.2 & $* * *$ & 7.4 & $* * *$ & 3.2 & $* * *$ \\
\hline Cunaxoides sp. nov. 2 & 4.1 & $*$ & 0 & n.s & 2.1 & $*$ & 19 & $* * *$ & 2 & $*$ & 3.8 & $* * *$ & 1.6 & n.s \\
\hline Pulaeus sp. nov. 9 & 2.8 & n.s. & 7.8 & n.s. & 3.4 & $* * *$ & 3.1 & $*$ & 2.3 & $*$ & 4.1 & $* * *$ & 3.3 & $* * *$ \\
\hline Pulaeus sp. nov. 14 & 0 & n.s. & 22 & $* * *$ & 2.2 & $*$ & 0 & n.s. & 1.9 & $*$ & 2.6 & $* *$ & 1.9 & $*$ \\
\hline Pulaeus sp. nov. 16 & 2.4 & n.s. & 13 & $* * *$ & 1.9 & $*$ & 11 & $* *$ & 1.8 & $*$ & 4.6 & $* * *$ & 0.9 & n.s. \\
\hline Neocunaxoides sp. nov. 1 & 0.7 & n.s & 4.6 & $*$ & 6.2 & $* * *$ & 1.5 & n.s & 2.3 & $* *$ & 5.1 & $* * *$ & 1.5 & n.s \\
\hline Pulaeus sp. nov. 1 & 0.1 & n.s. & 1.7 & n.s. & 4 & $* * *$ & 2.7 & n.s. & 4.9 & $* * *$ & 2.3 & $* *$ & 2.3 & $*$ \\
\hline Neoscirula sp. nov. 1 & 1 & n.s. & 6.2 & $*$ & 1.4 & n.s. & 1 & n.s. & 1.9 & $*$ & 1.4 & n.s. & 1.9 & $*$ \\
\hline Coleoscirus simplex & 0.8 & n.s. & 57 & $* * *$ & 3.1 & $* *$ & 1.3 & n.s. & 1.3 & n.s. & 2.4 & $* *$ & 0.5 & n.s. \\
\hline Cunaxa potchensis & 0.4 & n.s & 57 & $* * *$ & 3.3 & $* * *$ & 1.3 & n.s & 0.9 & n.s & 2.6 & $* *$ & 0.5 & n.s \\
\hline Dactyloscirus sp. nov. 1 & 1.5 & n.s & 27 & $* * *$ & 3 & $* *$ & 0.8 & n.s & 0.6 & n.s & 3.4 & $* * *$ & 0.7 & n.s \\
\hline Dactyloscirus sp. nov. 3 & 0.9 & n.s & 6.3 & $*$ & 3.1 & $* *$ & 3 & n.s & 1.6 & n.s & 2.5 & $* *$ & 1.2 & n.s \\
\hline Armascirus harrisoni & 1.8 & n.s & 18 & $* * *$ & 3.3 & $* * *$ & 0 & n.s & 0.5 & n.s & 2.2 & $*$ & 0.6 & n.s \\
\hline Pulaeus sp. nov. 7 & 0 & n.s & 2.4 & $*$ & 1.6 & $*$ & 0.3 & n.s & 2.1 & n.s & 1.9 & n.s & 2.1 & $* *$ \\
\hline Pulaeus sp. nov. 8 & 1.4 & n.s & 6.4 & $*$ & 1.9 & $*$ & 0.8 & n.s & 1 & n.s & 1.5 & n.s & 2.5 & $* *$ \\
\hline Pulaeus sp. nov. 12 & 8.5 & $* *$ & 0 & n.s. & 2.4 & $* *$ & 0 & n.s & 2.4 & $* *$ & 1.1 & n.s. & 1.1 & n.s \\
\hline Pulaeus sp. nov. 17 & 12 & $* *$ & 12 & $* *$ & 0.8 & n.s & 12 & $* *$ & 0.8 & n.s & 0.8 & n.s & 0.8 & n.s \\
\hline Neoscirula sp. nov. 2 & 0 & n.s & 8.6 & $* * *$ & 3.6 & $* * *$ & 0.4 & n.s & 1.3 & n.s & 1.3 & n.s & 1.5 & n.s \\
\hline Cunaxa sp. nov. 4 & 0.2 & n.s & 9.6 & $* *$ & 1 & n.s & 0.3 & n.s & 1.1 & n.s & 1.9 & $*$ & 1 & n.s \\
\hline Dactyloscirus sp. nov. 6 & 1.6 & n.s & 2.3 & n.s & 1.9 & $*$ & 0.2 & n.s & 0.3 & n.s & 2.2 & $*$ & 0.4 & n.s \\
\hline Pulaeus sp. nov. 5 & 0.5 & n.s & 0.6 & n.s & 2.4 & $* *$ & 0.4 & n.s & 0.5 & n.s & 2.7 & $* *$ & 0.5 & n.s \\
\hline Neoscirula sp. nov. 3 & 1.2 & n.s & 4.3 & $*$ & 1.4 & n.s & 1.2 & n.s & 1.7 & n.s & 1.4 & n.s & 1.7 & n.s \\
\hline Scutascirus sp. nov. 1 & 0 & n.s & 17 & $* * *$ & 1.1 & n.s & 0.8 & n.s & 1.4 & n.s & 0.9 & n.s & 1.5 & n.s \\
\hline Pseudobonzia sp. nov. 1 & 1.9 & n.s & 7.4 & $* *$ & 1.7 & n.s & 1.9 & n.s & 1.1 & n.s & 1.7 & n.s & 1.1 & n.s \\
\hline Cunaxa veracruzana & 3.7 & n.s & 3.7 & n.s & 2.6 & $* *$ & 2.7 & n.s & 1.3 & n.s & 1.3 & n.s & 2 & n.s \\
\hline Cunaxa sp. nov. 1 & 1.4 & n.s & 1.1 & n.s & 0.6 & n.s & 0.4 & n.s & 1.8 & n.s & 2.2 & $*$ & 1.2 & n.s \\
\hline Cunaxa sp. nov. 2 & 0.5 & n.s & 0 & n.s & 3.6 & $* * *$ & 0 & n.s & 1 & n.s & 1.8 & n.s & 0.8 & n.s \\
\hline Dactyloscirus sp. nov. 2 & 0.6 & n.s & 9.7 & $* *$ & 1.5 & n.s & 0.1 & n.s & 0.8 & n.s & 1.1 & n.s & 0.8 & n.s \\
\hline Cunaxoides sp. nov. 3 & 0 & n.s & 20 & $* * *$ & 0.7 & n.s & 0.2 & n.s & 1.7 & n.s & 1 & n.s & 1.2 & n.s \\
\hline Pulaeus pectinatus & 1 & n.s & 1.6 & n.s & 2.9 & $* *$ & 1.2 & n.s & 1.2 & n.s & 1.4 & n.s & 1.4 & n.s \\
\hline Pulaeus sp. nov. 10 & 0 & n.s & 0.5 & n.s & 2.3 & $*$ & 0.5 & n.s & 0.5 & n.s & 1.7 & n.s & 0.7 & n.s \\
\hline Pulaeus sp. nov. 11 & 4 & $*$ & 1 & n.s & 1.7 & n.s & 1 & n.s & 1.7 & n.s & 0.4 & n.s & 0.4 & n.s \\
\hline Pulaeus sp. nov. 13 & 0.5 & n.s & 6.5 & $*$ & 1.2 & n.s & 2.3 & n.s & 1.5 & n.s & 1.6 & n.s & 1.4 & n.s \\
\hline
\end{tabular}


CUADRO 4

Coeficiente de similitud faunistica de Sörensen entre las cuatro comunidades de cunáxidos

TABLE 4

Sörensen's similarity coefficient among four cunaxids communities

$\begin{array}{ccccc} & \mathrm{HC} 1 & \mathrm{HC} 4 & \mathrm{SC} 1 & \mathrm{SC} 4 \\ \mathrm{HC} 1 & 75 & 56 & 48 \\ \mathrm{HC} 4 & * & * & 58 & 57 \\ \mathrm{SC} 1 & * & * & * & 74 \\ \mathrm{SC} 4 & * & * & * & *\end{array}$

especies C. veracruzana y Cunaxa sp. nov. 1. (Fig. 5 y 6 ).

En las comunidades del suelo de febrero a junio fueron los meses más heterogéneos, ya que presentaron una baja equitativilidad y dominancia; con una alta diversidad, riqueza y densidad; las especies dominantes en ambas comunidades fueron: $C$. simplex, C. potchensis y Pulaeus sp. nov. 16 y sólo $C$. simplex en el SC4 (Fig. 5 y 6).

Por lo tanto, podemos concluir que las especies: C. simples, C. potchensis, Cunaxa sp. nov. 1, Cunaxa sp. nov. 2, C. veracruzana, Dactyloscirus sp. nov. 2, A. harrisoni, Cunaxoides sp. nov. 1, Cunaxoides sp. nov. 2, P. pectinatus, Pulaeus sp. nov. 14, Pulaeus sp. nov. 15 y Pulaeus sp. nov. 16 Scutascirus sp. nov.1 fueron las especies más representativas en el suelo y hojarasca de Chamela. Con respecto a la distribución en el transcurso del año, se observó que en la hojarasca de las dos cuencas, en febrero, fue en donde la mayoría de las especies presentaron una mayor densidad, mientras que en el suelo tuvo su incremento de febrero a junio en ambas cuencas; pero también el $\mathrm{SC} 1$ presentó un aumento en el número de ejemplares en diciembre y en el SC4 en octubre (Fig. 6).

Al estudiar la distribución de la densidad de las cuatro comunidades de cunáxidos en la época de lluvias (julio a noviembre) y sequía (diciembre a junio); en el suelo de ambas comunidades hubo un incremento en su densidad en la época seca, pero en la $\mathrm{HC} 1$ fue casi similar en ambos períodos; sin embargo, la HC4 fue la única comunidad que su densidad fue mayor en época de lluvias (Fig. 7). Las especies más abundantes en el suelo en la época seca fue $C$. potchensis y en la época húmeda lo fue $A$. harrisoni. Mientras que en la hojarasca en el período seco lo fue $A$. harrisoni y en el húmedo Cunaxoides sp. nov. 1. La especie que presentó una menor densidad en la hojarasca y suelo en ambos períodos fue Neoscirula sp. nov. 2 (Fig. 8).

\section{DISCUSIÓN}

De acuerdo con Martínez-Sánchez (1994), en la selva baja caducifolia en Chamela, los Cunaxidae ocupan el primer lugar en abundancia dentro de las familias de los Prostigmata (Cunaxidae, Eupodidae, Pachygnatidae (=Bimichaelidae), Nanorchestidae y Bdellidae). En nuestro estudio, los cunáxidos presentaron una abundancia total de 4720 individuos (3 325 ejemplares en el suelo, y 1395 en hojarasca). Comparando su abundancia con otro tipo de medios, en plantaciones de plátano en Brasil, donde se hizo un estudio de suelo orgánico durante 304 días, se observó una abundancia de 82 cunáxidos (Bettioll et al. 2002), ésto se debe a que son suelos manejados, y el área de nuestro estudio es un medio más estable y las condiciones son más adecuadas, y por lo tanto hay más disponibilidad de presas. Athias et al. (1974), encontraron que los bdélidos fueron más abundantes que los Cunaxidae en un ecosistema tropical húmedo en la sabana de Lamto. 

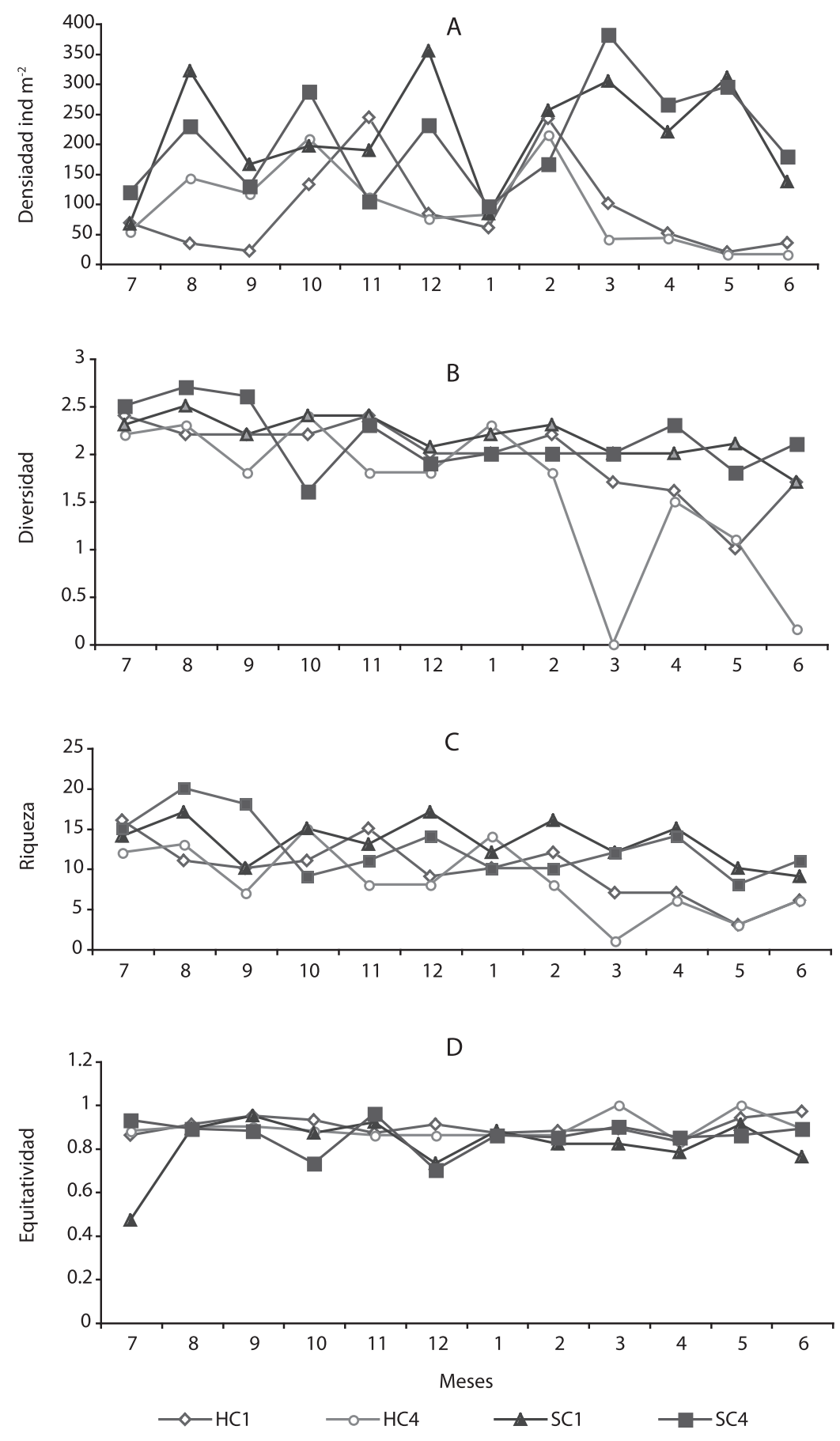

Fig. 5. Variación temporal en las cuatro comunidades de cunáxidos en la EBCH en Jalisco, México. A. Densidad (ind m-2); B. Indice de diversidad de Shanon (H'); C. riqueza; D. índice de Equitatividad de Pielou (J').

Fig. 5. Temporal variation in the four cunaxids communities in the $\mathrm{EBCH}$ in Jalisco, Mexico. A. Density (ind $\mathrm{m}^{-2}$ ); $\mathrm{B}$. Shanon's diversity index (H'); C. richness; D. Pielou's evenness index (J'). 

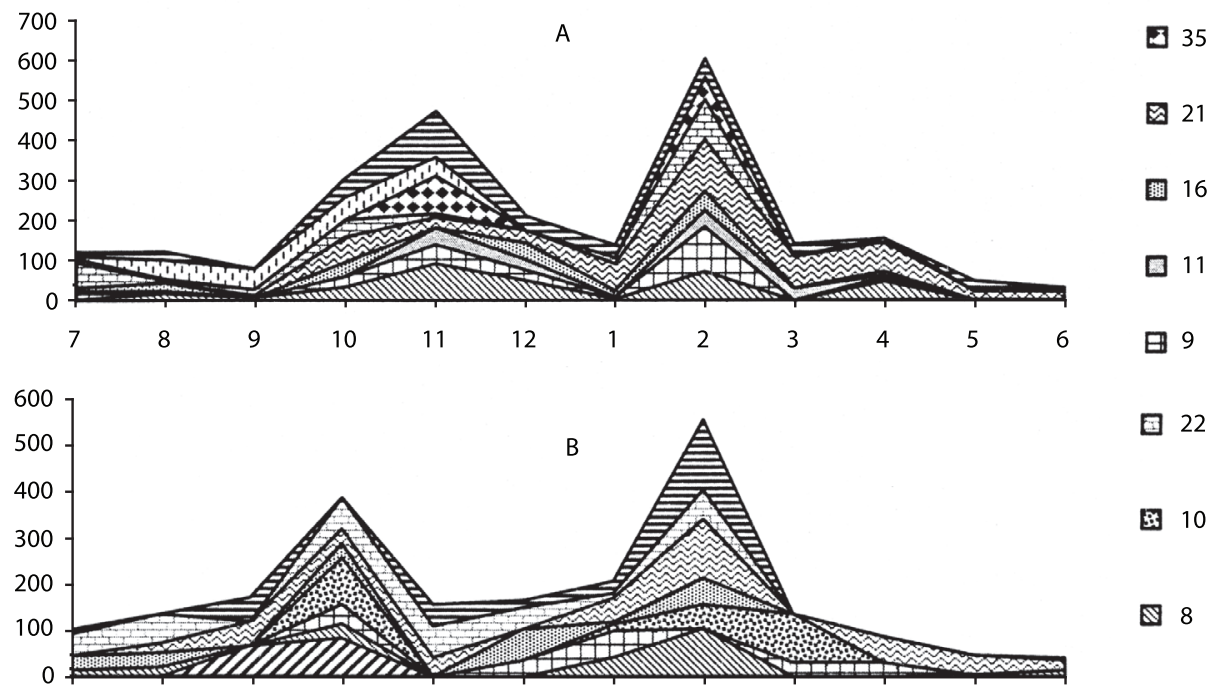

문 22

田 10

\$ 8

घ 5
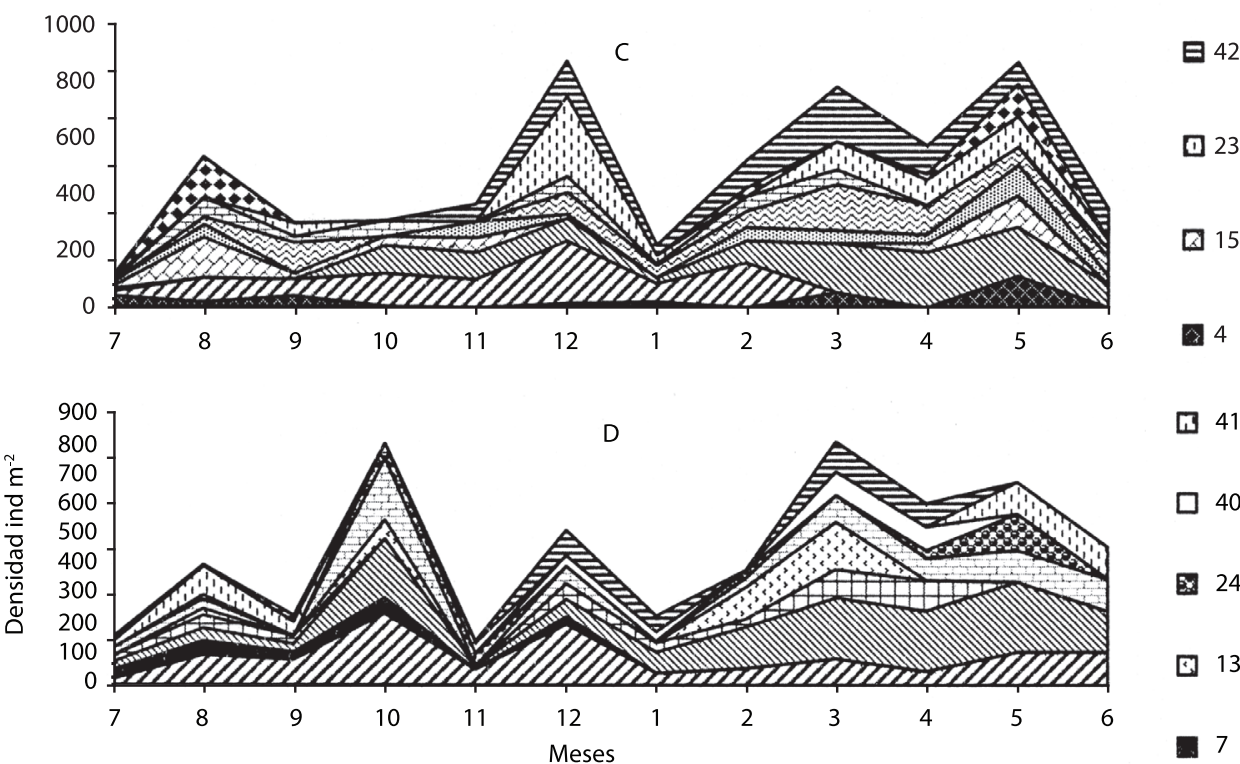

Fig. 6. Variación temporal de las especies más abundantes en las cuatro comunidades de EBCH en Chamela, Jalisco. A. Hojarasca cuenca 1; B. Hojarasca cuenca 4; C. Suelo cuenca 1; D. Suelo cuenca 4. 4=Scutascirus sp. nov. 1; 5=Coleoscirus simplex, 7=Pseudobonzia sp. nov. 2; 8=Cunaxa potchensis; 9=C. veracruzana; 10=Cunaxa sp. nov. 1; 11=Cunaxa sp. nov. 2; 13=Cunaxa sp. nov. 4; 15=Dactyloscirus sp. nov. 1; 16=Dactyloscirus sp. nov. 2; 21= Armascirus harrisoni; $22=$ Cunaxoides sp. nov. $1 ; 23=$ Cunaxoides sp. nov. 2; 24=Cunaxoides sp. nov. 3; 35=Pulaeus pectinatus; 40=Pulaeus sp. nov. $14 ; 41=$ Pulaeus sp. nov. 15; 42=Pulaeus sp. nov 16 .

Fig. 6. Temporal variation of the most abundant species in the four communities of EBCH in Chamela, Jalisco. A. litter watershed 1; B. litter watershed 4; C. soil watershed 1; D. soil watershed 4. 4=Scutascirus sp. nov. 1; 5=Coleoscirus simplex, $7=$ Pseudobonzia sp. nov. 2; 8=Cunaxa potchensis; 9=C. veracruzana; 10=Cunaxa sp. nov. 1; 11=Cunaxa sp. nov. 2; 13=Cunaxa sp. nov. 4; 15=Dactyloscirus sp. nov. 1; 16=Dactyloscirus sp. nov. 2; 21= Armascirus harrisoni; $22=$ Cunaxoides sp. nov. 1; 23=Cunaxoides sp. nov. 2; 24=Cunaxoides sp. nov. 3; 35=Pulaeus pectinatus; 40=Pulaeus sp. nov. 14; 41=Pulaeus sp. nov. 15; 42=Pulaeus sp. nov 16 . 


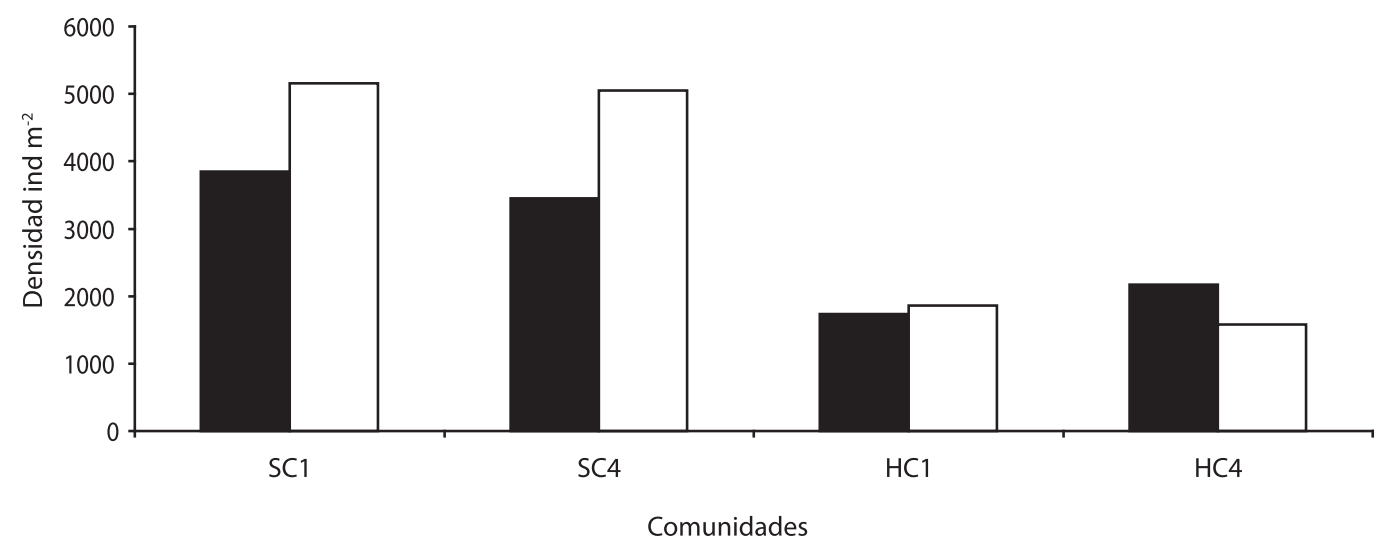

Epoca de lluvias

口Epoca seca

Fig. 7. Densidad total de las cuatro comunidades, agrupadas en la época seca y de lluvias en la selva baja caducifolia, Chamela, Jalisco.

Fig. 7. Total density of the four communities, in the dry and rainy periods, tropical dry forest, Chamela Biological Station, Jalisco.

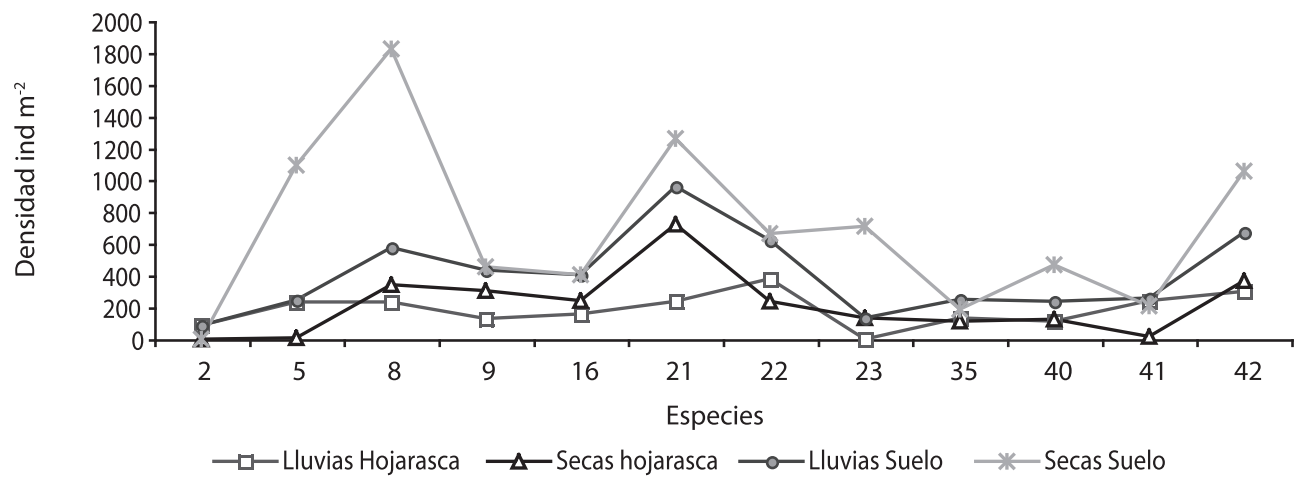

Fig. 8. Especies más abundantes en la época seca y húmeda en Chamela, Jal. 2=Neoscirula sp. nov. 2; 5=Coleoscirus simplex; 8=Cunaxa potchensis; 9=C. veracruzana; 16=Dactyloscirus sp. nov. 2; 21=Armascirus harrisoni; 22=Cunaxoides sp. nov. 1; 23=Cunaxoides sp. nov. 2; 35=Pulaeus pectinatus; 40=Pulaeus sp. nov. 14; 41=Pulaeus sp. nov. 15; 42=Pulaeus sp. nov 16.

Fig. 8. Species with the highest density in the dry and rainy seasons in Chamela, Jalisco. $2=$ Neoscirula sp. nov. 2; 5=Coleoscirus simplex; $8=$ Cunaxa potchensis; $9=$ C. veracruzana; 16=Dactyloscirus sp. nov. 2; 21=Armascirus harrisoni; $22=$ Cunaxoides sp. nov. 1;23=Cunaxoides sp. nov. 2; 35=Pulaeus pectinatus; $40=$ Pulaeus sp. nov. 14; 41=Pulaeus sp. nov. $15 ; 42=$ Pulaeus sp. nov 16.

También en un estudio realizado por Vázquez (2002) en una selva baja caducifolia, en la zona litoral de México, se cita una abundancia de 100 ejemplares de cunáxidos, en un muestreo que se hizo durante tres años por medio de trampas de pitfall. Estas diferencias tal vez se deban al tipo de vegetación y clima, el cual influye sobre las especies, así como el que tengan requerimientos ecológicos particulares y al tipo de muestreo. Otro factor puede ser que los organismos edáficos tienen la tendencia a formar poblaciones agregadas, lo que origina 
una variación en el número de especies dentro de una serie de muestras. En nuestra investigación, en algunas unidades de muestreo, los recuentos fueron muy bajos (inclusive cero) y en otras muy elevados como lo mencionan Astudillo et al. (1966).

Tomando en cuenta la estimación de la abundancia y densidad de los biotopos, las cuencas presentaron un comportamiento semejante. Además, es importante señalar que las comunidades de cunáxidos tuvieron un número mayor de organismos en el suelo, debido a que en las zonas estudiadas no se observó un verdadero extracto de hojarasca, por lo tanto los factores ambientales y biológicos actúan de una manera más drástica. Santos et al. (1981) consideran que un buen aporte de hojarasca sirve como un reservorio de nutrientes, el cual puede influir en la abundancia de los organismos. Cuando las condiciones microclimáticas son críticas, los organismos tiendan a adaptarse a un hábitat más estable (capas menos superficiales del suelo), como lo menciona Whitford (1984), y para protegerse de los cambios de temperatura suelen presentar movimientos verticales en el mantillo y los 20 primeros centímetros del suelo.

Medir la abundancia relativa y coeficiente de frecuencia de cada especie de cunáxidos, nos permitió identificar aquellas especies que por su escasa representatividad en la comunidad son más sensibles a las perturbaciones ambientales, o a otros factores. En este estudio el coeficiente de frecuencia todas las especies fue accidental (100\%), y su abundancia relativa tuvo un $30 \%$ de especies raras en ambas cuencas y biotopos; los ecólogos dan varias explicaciones a este comportamiento, ya que consideran que el reparto de los recursos, da como resultado que la abundancia de las especies, en algunos casos, es equivalente a la parte del espacio del nicho que ocupan o prefieren estás. Otro factor podría ser su diversidad temporal, como pueden ser las diferentes actividades diarias, ya que existe especies diurnas y nocturnas, que dan como resultado la presencia de especies "temporales", especies que no son residentes permanentes, y que están de paso, las cuales podría estar incrementando el número de especies en cada muestreo (Magurran 1989).

Las especies más dominantes de las comunidades de cunáxidos fueron: $C$. potchensis, A. harrisoni, Cunaxoides sp. nov. 1, Pulaeus sp. nov. 16, C. veracruzana, C. simples, Dactyloscirus sp. nov. 2 y Cunaxoides sp. nov. 2, tales especies presentan características morfológicas de los organismos hemiedáficos ó euedáficos, los cuales deben tener modificaciones para facilitar sus movimientos en el suelo, y en estos cunáxidos presentan el cuerpo pequeño y alargado (alargamiento del idiosoma entre el intervalo de las patas II y III) y poco plano, no esclerosado, con estriaciones, sin pigmentación, así como tener los pedipalpos y quelíceros cortos ó reducidos (tarsos) para poder pasar a través de suelo, sedas del cuerpo cortas, y las patas sus tarsos y tibias cortos y delgados, lo que los hacer tener movimientos rápidos (Rapoport 1970, Lindquist y Palacios-Vargas 1991, Villani et al. 1999). Además de lo anterior, se puede inferir que el clima estacional que presenta la zona de estudio, pudo provocar agregación de los cunáxidos en zonas con mejor condiciones, y el suelo es un medio más estable, en el cual los cambios climáticos no son tan drásticos como en la hojarasca, y por lo tanto, influyen indudablemente en su abundancia y riqueza.

Comparando el comportamiento de otros microartrópodos edáficos estudiados en la misma zona y biotopos, como son los colémbolos, con los datos obtenidos con cunáxidos, observamos que la Familia Isotomidae y Onychiridae coinciden en sus patrones de densidad con las especies $A$. harrisoni, $C$. simplex, Dactyloscirus sp. nov. 2, P. pectinatus, Pulaeus sp. nov. 15 y Pulaeus sp. nov. 16, C. veracruzana, Cunaxoides sp. nov. 2, C. potchensis y C. simplex (Gómez-Anaya 1998, Gómez-Anaya y Palacios-Vargas 2004). Por otro lado, en un estudio realizado por Walter y Kaplan (1991) sobre los hábitos alimenticios de los géneros Pulaeus, Cunaxa y Dactyloscirus, se encontró que se alimentan de algunos grupos de colémbolos (Isotomidae y Onychiridae), lo que nos hace suponer que el comportamiento 
sea similar en los colémbolos (presas) y los cunáxidos (depredadores), y que cuando hay mayor disponibilidad de recursos y factores abióticos (humedad, temperatura, porosidad, materia orgánica) en una sitio determinado, esto propicia la agregación de las individuos

En las comunidades del suelo las especie más importantes fueron: C. simplex, C. potchensis, A. harrisoni, Cunaxoides sp. nov. 1 y Pulaeus sp. nov. 16, probablemente estas especies se encuentren cumpliendo un papel ecológico específico, modificando la densidad de las poblaciones de los cunáxidos, dentro de este ecosistema (selva baja caducufolia), y sean unas especies generalistas eficientes las cuales utilicen los recursos intensamente, y al existir una gran competencia, desplacen a las especies menos abundantes y competitivas.

La variación temporal en las comunidades de los cunáxidos presentó un comportamiento distinto, ésto se debe a que los factores abióticos (temperatura, precipitación, humedad y composición del suelo y cobertura vegetal) y los bióticos (competencia y depredación), se encuentran estrechamente relacionados con la abundancia y distribución de los organismos. Los resultados durante el desarrollo de esta investigación, al analizar la densidad que presentan las cuencas y biotopos, nos permiten señalar que ambos siguen una conducta semejante, el suelo sobresalió en abundancia, presentando dos picos máximos, uno después de la época húmeda y el otro después de la lluvia atípica de enero. Mientras que en la hojarasca se presentaron en febrero, noviembre. El comportamiento de los cunáxidos fue semejante al que en general se presenta en los ácaros y en particular los Prostigmata, ya que su densidad incrementa después de los períodos de lluvia (Luxton 1981). Quizá también se relacione con otros factores, como pueden ser la densidad de otros depredadores y la cantidad de presas disponibles, como los son los colémbolos, oribátidos, nemátodos y Tydeidae (Walter y Kaplan 1991). Un comportamiento contrario se observó con las especies de bdélidos (Mejía-Recamier 1997), ésto tal vez se deba a que existe una competencia más intensa por el alimento y espacio entre los miembros de la comunidad (intraespecífica ó interespecífica) en esta época, lo que determina la abundancia de estos organismos y la Familia Cunáxidae sea un depredador más eficiente en este medio.

Tomando en cuenta sólo a los biotopos, en la hojarasca los índices de diversidad, equidad y riqueza disminuyeron. En general la hojarasca se considera como un microhábitat temporal e inestable, el cual sustenta una gran variedad de especies accidentales y raras. De acuerdo a lo mencionado por Coddington et al. (1996), las especies raras incrementan la estimación de la riqueza, pero no son miembros permanentes de las comunidades que están siendo estimadas. Por otro lado, en el suelo dominan un número menor de especies, y puede deberse a que sólo algunas especies se especializan a un biotopo determinado, ó si las condiciones son mejores, debido al aumento de producción de frutos, flores y de hojarasca, se provoca que tengan movimientos tanto verticales y como horizontales. Como los cunáxidos son depredadores muy activos, tienden a buscar presas en lugares en donde hay buenas condiciones, como puede ser el suelo y la hojarasca.

El índice de diversidad y riqueza anual que se observó en las diferentes comunidades de cunáxidos, revela que fue constante la mayor parte del año, pero abril desciende en la HC4, esto lo podemos asociar con los factores abióticos como la precipitación atípica, que fue la más elevada que se ha citado en esta zona, además el tamaño y contenido de hojarasca que fue el mes con un menor contenido, las cuales afectaron de cierta forma a las comunidades. Se ha observado que cuando se presentan situaciones extremas de perturbación, así como escasez de recursos, da como resultado una disminución de la diversidad y riqueza.

El índice de similitud que se obtuvo en las cuatro comunidades reflejan una elevada similitud entre las comunidades, las que presentaron el más alto índice de similitud fueron el SC1 y SC4 con la $\mathrm{HC} 1$ y $\mathrm{HC} 4(75 \%)$, esto tal vez se deba a que tales comunidades presentaron semejante equidad, diversidad y riqueza, lo que hace que presenten una alta similitud. 
La actividad de los depredadores es esencial para el buen funcionamiento de todos los ecosistemas terrestres, por lo tanto los cunáxidos juegan un papel importante dentro de los distintos ecosistemas ya que intervienen en la regulación de las poblaciones. Por lo que es necesario conocer la estructura de las comunidades de los depredadores benéficos, para poder establecer las relaciones entre la estructura y fenología vegetal.

Sus funciones dentro de las cadenas tróficas, pueden ser las de suprimir en las poblaciones a los individuos no adaptados o enfermos, así como prevenir la superpoblación, ya que una población que no esta regulada eficazmente por una serie de factores externos, puede transformarse en plaga. Sin embargo, por lo común existe un equilibrio de las poblaciones naturales, en el cual juegan un papel decisivo los depredadores. Los cunáxidos, en particular, regulan las poblaciones de algunos colémbolos y ácaros que causan daños a cultivos, por lo que se les considera como eficaces en el control biológico (Muma 1960, Schruft 1971, Kethley 1982, Tawfik 1984, May 2001).

El presente trabajo constituye uno de los pocos enfocados hacia el estudio de los patrones de distribución espacial y temporal de los cunáxidos en suelos, y el gran número de especies nuevas encontradas, que actualmente se encuentran en estudio (Mejía-Recamier y Palacios-Vargas, en prep.), pone en evidencia la diversidad del grupo y su importancia faunística y ecológica en los suelos de México.

\section{AGRADECIMIENTOS}

El presente trabajo fue realizado con material obtenido del proyecto DGAPA IN-7820/20 "Efectos del uso de una selva baja caducifolia sobre la fauna de artrópodos". Agradecemos a José Antonio Gómez Anaya y Alicia Rodríguez Palafox por la recolecta y procesamiento de las muestras de suelo y hojarasca. Este trabajo está dedicado a la memoria de Alicia Rodríguez Palafox.

\section{RESUMEN}

Se estudió la variación espacial y temporal de la estructura de la comunidad de los cunáxidos edáficos de una selva baja caducifolia en Chamela, Jalisco, México. Se tomaron muestras mensuales de suelo y hojarasca durante un año (Julio 1991-Junio 1992) en dos cuencas. Registramos 43 especies, para las cuales se proporciona su abundancia, frecuencia, abundancia relativa, riqueza, densidad, diversidad, equitatividad y rareza. El número de especies fue similar en el suelo y la hojarasca, pero ambos biotopos difirieron en su composición. La mayor densidad se encontró en el suelo con 5074 ind $\mathrm{m}^{-2}$. Pulaeus fue el género que presentó la mayor riqueza. De acuerdo a la permanencia y abundancia relativa, las especies dominantes, tanto en el suelo y como en la hojarasca, fueron: Colescirus simples, Cunaxa potchensis, Armascirus harrisoni y Pulaeus sp. nov. 16. El suelo presentó una mayor dominancia y densidad, siendo C. potchensis, C. simplex, A. harrisoni Cunaxoides sp. nov. 1 y Pulaeus sp. nov. 16 las especies más abundantes. En la hojarasca se registró una mayor variación temporal en cuanto a densidad, diversidad y riqueza entre abril a junio, y en el suelo de septiembre a noviembre.

Palabras clave: cunáxidos, diversidad, riqueza, variación estacional, suelo, hojarasca.

\section{REFERENCIAS}

Athias, F., G. Josens, P. Lavelle \& R. Schaefer. 1974. Les microarthropodes du sol de la Savane de Lamto. Bull. Liaison Chercheurs de Lamto 5: 55-89.

Astudillo, V.M., A. Morales \& R. Loyola. 1966. Problemas en el análisis estadístico de poblaciones con distribución contagiosa. Programa en Biología del Suelo. 1er. Coloquio Latinoamericano de Biología del Suelo, UNESCO, México DF: 359-369.

Baker, E.W. \& A. Hoffmann. 1948. Ácaros de la familia Cunaxidae. An. Esc. Nac. de Cienc. Biol. Méx. 5: 229-273.

Bettiol1 W., R. Ghini, J.A. Haddad, M.A. Vieira. 2002. Soil organisms in organic and conventional cropping systems. Sci. Agric. 59: 565-572.

Bullock, S.H. 1986. Climate of Chamela, Jalisco and trends in south coastal region of Mexico. Arch. Met. Geoph. Biocl. 36: 297-316.

Bullock, S.H. 1988. Rasgos del ambiente físico y biológico de Chamela, Jalisco, México. Folia Entomol. Mex. 77: 5-17. 
Cervantes, S.L., M. Maass \& R. Domínguez. 1988. Relación lluvia- escurrimiento en un sistema pequeño de cuencas de Selva Baja Caducifolia. Ingeniería Hidráulica en México 3: 24-30.

Coddington, J.A., L.H. Young \& F.A. Coyle. 1996. Estimating spider species richness in a Southern Appalachian cave hardwood forest. J. Arac. 24: 111-128.

Cotler, H., E. Durán \& Ch. Siebe. 2002. Caracterización morfo-edafológica y calidad de sitio de un bosque tropical caducifolio, p. 17-79. In F.A. Noguera, J.H. Vega R., A.N. García A. \& M. Quezada A. (eds). Historia Natural de Chamela, Instituto de Biología, UNAM, México.

De la Garza, J. 2003. Efecto de las prácticas sobre la mesofauna edáfica con énfasis en Collembola. Tesis Profesional, Universidad Nacional Autónoma México, D.F., México.

Estrada, E.G. \& I. Sánchez. 1986. Ácaros del suelo de dos zonas del Valle de Tehuacán, Puebla. Tesis Profesional. ENEP. Iztacala, Universidad Nacional Autónoma México, D.F., México.

García, E. 1988. Modificaciones al sistema de clasificación climática de Koppen. Instituto de Geografía, Universidad Nacional Autónoma de México, D.F., México.

García-Oliva, F., A. Camou \& J.M. Maass. 2002. El clima de la región central de la costa del Pacifico mexicano, p. 3-10. In F.A. Noguera, J.H. Vega R., A.N. García A \& M. Quezada A. (eds). Historia Natural de Chamela, Instituto de Biología, UNAM, México.

Gómez-Anaya, J.A. 1998. Ecología de Collembola (Hexapoda: Apterygota) de Chamela, Jalisco, México. Tesis de Maestría, Universidad Nacional Autónoma México, D.F., México.

Gómez-Anaya, J.A. \& J.G. Palacios-Vargas. 2004. Structure and composition of litter and soil Poduromorpha assemblages (Hexapoda: Entognatha: Collembola) from a tropical dry forest in western Mexico. Folia Entomol. Mex. 43: 215-225.

Kethley, J. 1990. Acarina: Prostigmata (Actinedida), p. 667-756. In D.L. Dindal (ed). Soil Biology Guide. Wiley, Nueva York, EEUU.

Kethley, J. 1982. Acariformes, p. 117-146. In S.P Baker (ed). Sypnopsis and clasification of living organisms. McGraw-Hill, Nueva York, USA.
Lindquist, E.E. \& J.G. Palacios-Vargas. 1991. Proterorhagiidae (Acari: Endeostigmata), a new Family of Rhagidid-like mites from Mexico. Acarol. 32: 341-363.

Lott, E.J. \& T.H. Atkinson. 2002. Biodiversidad y fitogeografía de Chamela-Cuixmala, Jalisco, p. 83-97. In F.A. Noguera, J.H. Vega R., A.N. García A. \& M. Quezada A. (eds). Historia Natural de Chamela, Instituto de Biología, UNAM, México.

Ludwing, J. \& J. Reynolds. 1988. Statistical Ecology. A primer on methods and Computing. Wiley-Intercience, Nueva York, EEUU.

Luxton, M. 1981. Studies on the prostigmatic mites of a Danish beech wood. Pedobiologia 23: 1-8.

Magurran, A.E. 1989. Ecological Diversity and its Measurement. Princeton University, Nueva Jersey, EEUU.

Martínez-Yrízar, A., J.M. Maass, A. Pérez-Jiménez \& J. Sarukhán. 1996. Net primary productivity of a tropical deciduous forest ecosystem in western Mexico. J. Trop. Ecol. 12: 169-175.

Martínez-Sánchez, J. 1994. Análisis cuantitativo de los ácaros Prostigmata edáficos de la selva de Chamela, Jalisco. In Sociedad Mexicana de Entomología. Resumen XXIX. Congreso Nacional de Entomología, México DF, México.

Mejía-Recamier, B.E. 1997. Aspectos ecológicos de los ácaros de la Familia Bdellidae de Chamela, Jalisco. Tesis de Maestría, Universidad Nacional Autónoma México, D.F., México.

Michocka, S. 1987. Polskie roztocze (Acari) Zrodzin Bdellidae I. Cunaxidae. Monogr. faun. Pol. 14: 1-120.

Moreno-Moreno, J.A. 1996. Soil mites from the high altitude pine forest in Central Mexico, p. 579-584. In R. Mitchell, D.J. Horn, G.R. Needham \& W.C. Welbourn (eds). Acarology IX, Proceedings. Ohio Biological Survey, Columbus, Ohio.

Muma, M.H. 1960. Predatory of the Family Cunaxidae associated with citrus in Florida. Ann. Entomol. Soc. Amer. 53: 321-326.

Patiño, M.C. 1990. Variación espacial y temporal de la capa de hojarasca (mantillo) en una selva baja caducifolia en Chamela, Jal. México. Tesis Profesional, Universidad Nacional Autónoma México, D.F., México. 
Rapoport, E.H. 1970. Fauna del suelo: Algunos datos sobre su abundancia y distribución, p. 469-481. In La Ciencia en Venezuela. Universidad de Carabobo, Carabobo, Venezuela.

Santos, P.F. \& W.G. Whitford. 1981. The effects of microarthropods in litter decomposition in a Chihuahuan desert ecosystem. Ecology 62: 654-663.

Sorensen, J.T., D.N. Kinn \& R.L. Doutt. 1983. Biological observations on Bdella longicornis: A predatory mites in California vineyards (Acari: Bdellidae). Entomonogr. 2: 297-305.

Schruft, G. 1971. Haleupalus oliveri new species, A "Thorn-palped" mites on grape vines (Vitis Spp) Acari: Cunaxidae. Deut. Entomol. Zeits. 18: 379382.

Smiley, R.L. 1992. The predatory mite family Cunaxidae (Acari) of the world with a new classification. Indira Publising House, Michigan, EEUU.

StatSoft Inc. 1995. Statical user guide. Complete Statistical System StatSsft. Oklahoma

Van Straalen, N.M. \& H.A. Verhoef. 1997. The development of a bioindicador system for soil acidity based on arthropod pH referents. J. Appl. Ecol. 34: 217-232.

Vázquez Rojas, I.M. 2002. Prostigmata (Acárida) edáficos de una zona litoral del Golfo de México. Tesis de
Doctorado, Universidad Nacional Autónoma México, D.F. México.

Villani, M.G., L.L. Alle, A. Díaz \& P.S. Robbins. 1999 Adaptative strategies of edaphic arthropods. Ann. Rev. Entomol. 44: 233-256.

Walter, D.E. \& D.T. Kaplan. 1991. Observation on Colescirus simplex (Acarina: Prostigmata), a predatory mites colonizer greenhouse cultures of rootknot nematodes (Meloidogyne Spp) and review of feeding behavior in the Cunaxidae. Exp. Appl. Acarol. 12: 47-59.

Wallwork, J.A., B.W. Kawill \& W.G. Whitford. 1985. Distribution and diversity patterns of soil mites and another microarthropods in a Chihuahua desert sites. J. Arid Environ. 9: 215-231.

Whitford, W.G. 1984. The effects of high salt concentration on desert soil microarthropod density and diversity. Natur. 29: 239-242.

Zar, H.J. 1984.Biosstatistica análisis. Printice may, Englewood Cliffs, Nueva Jersey, EEUU.

\section{REFERNCIA DE INTERNET}

May, J. 2001. Citrus red mites in Arizona. University Arizona Cooperative Extension p://ag.arizona.edu/ yuma/urbanhorticulture/citrusredmite.htm (consultado 12 mayo 2007). 